\title{
Obtención, caracterización y evaluación in vitro de recubrimientos de policaprolactona-quitosano sobre la aleación Ti6Al4V tratada químicamente
}

\author{
Liliana S. Gómez Rodríguez ${ }^{\mathrm{a}, \bigotimes}$, Andrés F. Quintero Jaime ${ }^{\mathrm{a}}$, \\ Darío Y. Peña Ballesteros ${ }^{\mathrm{a}}$, Hugo A. Estupiñan Durán ${ }^{\mathrm{b}}$ \\ ${ }^{a}$ Escuela de Ingeniería Metalúrgica, Universidad Industrial de Santander, Carrera 27 calle 9 Ciudad \\ Universitaria, Bucaramanga-Santander, Colombia \\ ${ }^{b}$ Facultad de Minas, Universidad Nacional de Colombia, Medellín-Antioquia, Colombia \\ Autor para la correspondencia: inglilisofi@gmail.com
}

Enviado: 1 Octubre 2013; Aceptado: 11 Abril 2014; Publicado on-line: 08 September 2014

\begin{abstract}
RESUMEN: Se obtuvieron recubrimientos poliméricos de policaprolactona-quitosano, aplicados mediante la técnica Dip-Coating, sobre sustratos de Ti6Al4V tratados químicamente con $\mathrm{NaOH}$. Con base en los análisis SEM y los espectros infrarrojos, se observó que la cantidad de policaprolactona en el recubrimiento presentó un efecto en la retención de quitosano en la superficie, relacionado con la emisión del enlace R-OH y las características morfológicas. Los espectros de impedancia realizados a las películas poliméricas, mostraron procesos relacionados con fenómenos de adsorción de especies iónicas hasta la formación de monocapas en la superficie. Igualmente, estos espectros mostraron fenómenos de transferencia de carga generados por las características morfológicas de los recubrimientos, como sus porosidades, densidad y homogeneidad. La inmersión de los sustratos recubiertos en una solución SBF durante 8 días, permitió observar su capacidad de adsorción de calcio mediante la nucleación y precipitación de fosfatos de calcio, mostrando un carácter bioactivo frente al medio.
\end{abstract}

PALABRAS CLAVE: Bioactividad; Fosfato de calcio; Policaprolactona; Quitosano; Ti6Al4V

Citation / Cómo citar este artículo: Gómez, L., Quintero, A., Peña, D., Estupiñan, H. (2014) "Obtención, caracterización y evaluación in vitro de recubrimientos de policaprolactona-quitosano sobre la aleación Ti6Al4V tratada químicamente". Rev. Metal. 50(3): e021. doi: http://dx.doi.org/10.3989/revmetalm.021

ABSTRACT: Obtention, characterization and in vitro evaluation of polycaprolactone-chitosan coatings growth on chemically treated Ti6Al4V alloy. Polymeric coatings were obtained polycaprolactone-chitosan. The coatings were applied by dip-coating technique, on Ti6Al4V substrates chemically treated with $\mathrm{NaOH}$ solution. Based on SEM morphological analysis and infrared spectra, it was observed that the amount of polycaprolactone in the coating obtained had an effect in retaining the chitosan on the surface, associated with the emission of $\mathrm{R}-\mathrm{OH}$ bond and the morphological characteristics. Impedance spectra performed on the polymeric films showed phenomena related processes adsorption of ionic species to monolayer formation on the surface. These spectra showed equally charge transfer phenomena generated by the morphological characteristics of the coatings, such as its porosity, density and homogeneity. The coated substrate was immersing in SBF solution for 8 days, allowed to observe its adsorption capacity of calcium through nucleation and precipitation of calcium phosphates, bioactive character displaying a front medium.

KEYWORDS: Bioactivity; Chitosan; Phosphate of calcium; Polycaprolactone; Ti6A14V

Copyright: (C) 2014 CSIC. This is an open-access article distributed under the terms of the Creative Commons Attribution-Non Commercial (by-nc) Spain 3.0 License. 


\section{INTRODUCCIÓN}

El titanio y sus aleaciones son por excelencia los biomateriales metálicos más empleados para la sustitución y regeneración de tejidos óseos, debido a sus excelentes propiedades; sin embargo, una elevada resistencia a la corrosión le confiere una inercia química al titanio que no permite una adecuada interacción con el medio biológico, dificultando el proceso de osteointegración (Bronzino, 2000).

Múltiples investigaciones se han llevado a cabo en los últimos años con el propósito de modificar la superficie del titanio y sus aleaciones, debido a que en gran medida el proceso de osteosíntesis se ve ampliamente influenciado por las reacciones e interacciones que ocurren en la interfase formada entre la superficie del implante y el medio biológico.

En este orden de ideas, el desarrollo de técnicas como el tratamiento superficial con soluciones químicas, la oxidación anódica, el uso de recubrimientos cerámicos y/o poliméricos, la implantación iónica, la modificación de la rugosidad mediante micro y nano indentación, entre otras, permiten activar químicamente la superficie del biomaterial frente al medio biológico, lo cual genera las condiciones adecuadas para garantizar el proceso de osteointegración (Xuanyong et al., 2004; Peón et al., 2005; Multigner et al., 2009; Paz et al., 2011; Tojal et al., 2013).

Entre las técnicas más empleadas se encuentra el tratamiento con soluciones de $\mathrm{NaOH}$ o $\mathrm{KOH}$, debido a que ocurre una incorporación de iones hidróxido (OH-) que actúan como sitios preferenciales en nucleación de fosfatos de calcio, específicamente hidroxiapatita $\mathrm{Ca}_{5}\left(\mathrm{PO}_{4}\right)_{3}(\mathrm{OH})$. Así mismo el uso de recubrimientos poliméricos biodegradables como el ácido poliláctico (PLA), ácido poliglicólico (PGA), policaprolactona (PCL), colágeno y quitosano, los cuales durante su proceso de degradación liberan sustancias metabolizables por las células, promueven condiciones óptimas para su crecimiento y proliferación celular (Estupiñan, 2011).

\section{MATERIALES Y MÉTODOS}

\subsection{Materiales}

Los tratamientos con soluciones fisiológicas y los recubrimientos fueron realizados sobre muestras cilíndricas de Ti6Al4V de $14 \mathrm{~mm}$ de diámetro y $2 \mathrm{~mm}$ de altura. Para la obtención de los recubrimientos biopoliméricos se utilizó quitosano con $85 \%$ de desacetilación y policaprolactona (PCL) marca Sigma-Aldrich, con una masa molecular promedio $\left(\mathrm{M}_{\mathrm{n}}\right)$ de 80.000 .

\subsection{Preparación de materiales}

\subsubsection{Preparación superficial de la aleación Ti6 Al4V}

Las muestras de Ti6Al4V fueron pulidas empleando papel abrasivo de $\mathrm{SiC}$ y suspensiones de alúmina con un tamaño de partícula promedio de 0,5 y $0,05 \mu \mathrm{m}$. Posteriormente las muestras pulidas fueron decapadas, sumergiéndolas en una solución acuosa $3 \%$ v/v de $\mathrm{HF}$ y $20 \%$ v/v de $\mathrm{HNO}_{3}$ durante 5 minutos.

\subsubsection{Tratamiento alcalino}

El tratamiento alcalino se llevó a cabo sumergiendo las muestras pulidas y decapadas de Ti6Al4V en una solución acuosa $10 \mathrm{M}$ de $\mathrm{NaOH}$ a $60^{\circ} \mathrm{C}$ durante 24 horas. Al finalizar el tratamiento, las muestras fueron lavadas y secadas durante 24 horas a temperatura ambiente dentro de un desecador (Kim et al., 1997). Con el fin de estabilizar el sólido formado, se realizó un tratamiento térmico con una rampa de calentamiento de $25^{\circ} \mathrm{C}$ a $450{ }^{\circ} \mathrm{C}$ en 43 minutos, manteniendo esta temperatura durante 1 hora.

\subsubsection{Obtención de soluciones de PCL-Quitosano}

Las soluciones de PCL-Quitosano fueron preparadas a partir de soluciones individuales de PCL y quitosano. El quitosano fue disuelto en una solución acuosa $0,5 \mathrm{M}$ de ácido acético durante 1 hora a $500 \mathrm{rpm}$, obteniendo una solución de quitosano $1 \%$ w/v. Simultáneamente la PCL fue disuelta en ácido acético glacial durante 2 horas a $1000 \mathrm{rpm}$. Las soluciones individuales de PCL y quitosano fueron mezcladas manteniendo una relación volumétrica PCL/Quitosano de 10/3 a 1000 rpm durante 1 hora, hasta obtener soluciones estables (Reddy, 2011). Las cantidades de PCL se variaron con el fin de obtener mezclas poliméricas con relaciones $30-70,50-50$ y $70-30$.

\subsubsection{Conformación de los recubrimientos de PCL- Quitosano}

A partir de las soluciones estables de PCLQuitosano se realizó la conformación de los recubrimientos empleando la técnica de Dip-Coating. Las muestras de Ti6A14V tratadas fueron sumergidas en las diferentes soluciones poliméricas a una velocidad de descenso de $5 \mathrm{~cm} \mathrm{~min}^{-1}$, manteniéndolas en inmersión durante 10 segundos, para un posterior proceso de secado a $55{ }^{\circ} \mathrm{C}$ durante 30 minutos. El procedimiento de inmersión y extracción se realizó 3 veces con el fin de depositar 3 capas consecutivas, cada una conformada después del proceso de secado. 


\subsection{Caracterización}

\subsubsection{Microscopía electrónica de barrido (SEM)}

Las muestras poliméricas se recubrieron con oro y se estudió su morfología mediante microscopía electrónica de barrido utilizando un microscopio Quanta FEG 650, con una aceleración de electrones entre $5-10 \mathrm{kV}$.

\subsubsection{Espectroscopia infrarroja (FTIR)}

Las muestras biocerámico/biopolímero fueron estudiadas mediante espectroscopía de infrarrojo por transformada de Fourier (FTIR) utilizando un espectrofotómetro Shimadzu 8400S Fourier Transform.

\subsubsection{Inmersión de los recubrimientos PCL- Quitosano en SBF}

La capacidad de adsorción y precipitación del calcio se llevó a cabo mediante inmersión de las muestras recubiertas con la mezcla polimérica en un fluido fisiológico simulado (SBF) cuya composición se recoge en la Tabla 1 (Kim et al., 1997). Las superficies recubiertas se mantuvieron inmersas en el fluido simulado durante 8 días, manteniendo unas condiciones de temperatura constante de $36,5^{\circ} \mathrm{C}$.

\subsubsection{Espectroscopía de impedancia electroquímica}

Para la caracterización electroquímica de los recubrimientos se empleó una celda electrolítica plana con 3 electrodos conectada a un potenciostato-galvanostato Gamry 600. Se utilizó un electrodo de grafito como contraelectrodo, un electrodo de referencia Calomel saturado $3 \mathrm{M} \mathrm{KCl}$ y como electrodo de trabajo, la muestra de Ti6Al4V tratada y recubierta con la mezcla polimérica. El área de exposición con el electrolito fue de $1 \mathrm{~cm}^{2}$. Se aplicó un potencial de perturbación de $\pm 10 \mathrm{mV}$, realizando un barrido de frecuencias entre los 0,01 a $50.000 \mathrm{~Hz}$ a una temperatura de $25^{\circ} \mathrm{C}$.

\section{RESULTADOS Y DISCUSIÓN}

\subsection{Caracterización de la aleación Ti6Al4V}

La solución ácida utilizada en el decapado, atacó preferencialmente la fase beta de carácter dura y frágil, que se encuentra de forma globular y precipitó sobre la fase alfa blanda y dúctil, lo cual corresponde a las características microestructurales de las aleaciones de titanio bifásicas $\alpha+\beta$. En la Figura 1a se observa una superficie inicial homogénea, densa y libre de porosidades correspondiente a la formación de una capa de óxido de titanio que, después del tratamiento alcalino, sufrió una modificación morfológica al presentar la formación de una estructura granular, como se observa en la Figura 1b, tras el ataque de los iones hidroxilo $(\mathrm{OH}-)$ provenientes de la solución de $\mathrm{NaOH}$. Este proceso permitió la formación de un titanato de sodio $\mathrm{Na}_{2} \mathrm{Ti}_{5} \mathrm{O}_{11}$ por hidrolización del óxido. Tras el tratamiento térmico a $450{ }^{\circ} \mathrm{C}$ se produjo un cambio en la densidad de granos de la estructura, como se aprecia en la Figura 1c, atribuido al proceso de sinterización que presentó el cerámico.

\subsection{Caracterización de los recubrimientos PCL- Quitosano}

\subsubsection{Caracterización morfológica por microscopía SEM}

En la Figura 2a se aprecia la morfología de los recubrimientos PCL-Quitosano con una relación $30 \%$ y $70 \%$, respectivamente. Dichos recubrimientos muestran, principalmente, una superficie heterogénea, compuesta por zonas parcialmente recubiertas, que ponen de manifiesto la estructura granular del titanato de sodio obtenido tras el tratamiento alcalino, sugiriendo un bajo espesor de la película formada. De igual forma, en esta clase de recubrimientos se observó la formación de burbujas en su superficie, causadas por gas atrapado durante el proceso de secado del solvente.

La Figura 2b muestra las micrografías SEM correspondientes a los recubrimientos con relación $50 \%$ PCL y 50\% quitosano. Estos recubrimientos indican una escasa homogeneidad superficial, puesto que se observa la presencia de zonas compuestas por una matriz de quitosano, con una morfología porosa, acompañada de precipitados de fases poliméricas de PCL en su interior. En ciertas zonas de la superficie se observan rugosidades debidas a la formación de una película homogénea y continua, que cubre la matriz porosa de quitosano.

La precipitación de fases de PCL en la matriz porosa y la formación de una película continua sobre la matriz de quitosano son ocasionadas durante el proceso de evaporación del solvente en la conformación de las películas, ya que el quitosano precipita primero, debido a su insolubilidad en el ácido acético glacial. En dicho proceso la policaprolactona

TABLA 1. Concentración de iones del fluido SBF expresada en mM

\begin{tabular}{lcccccccc}
\hline Elementos & $\mathbf{N a}^{+}$ & $\mathbf{K}^{+}$ & $\mathbf{M g}^{2+}$ & $\mathbf{C a}^{2+}$ & $\mathbf{C l}^{-}$ & $\mathbf{H C O}_{3}^{-}$ & $\mathbf{H P O}_{4}^{2-}$ & $\mathbf{S O}_{4}^{2-}$ \\
\hline Concentración & 142,0 & 5,0 & 1,5 & 2,5 & 147,8 & 4,2 & 1,0 & 0,5 \\
\hline
\end{tabular}



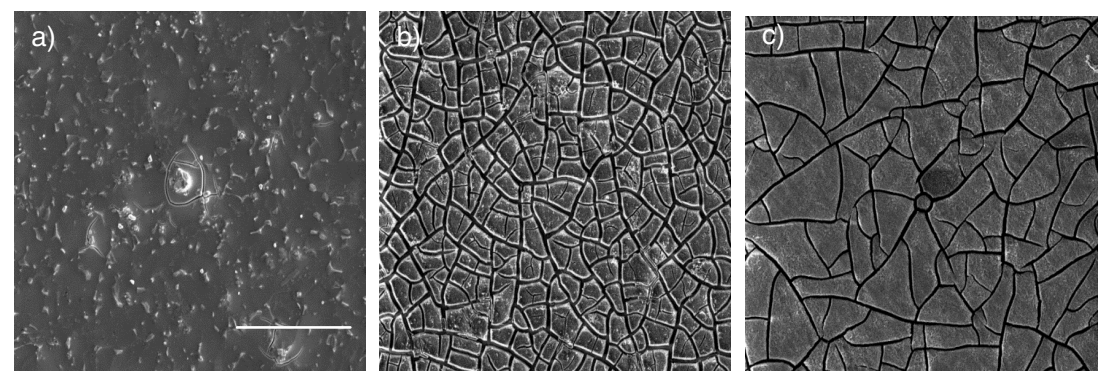

FIgURA 1. Micrografías SEM de la aleación Ti6Al4V: a) muestra sin tratar en solución alcalina, b) tratada en solución alcalina sin tratar térmicamente y c) tratada en solución alcalina y tratada térmicamente a $450{ }^{\circ} \mathrm{C}$.
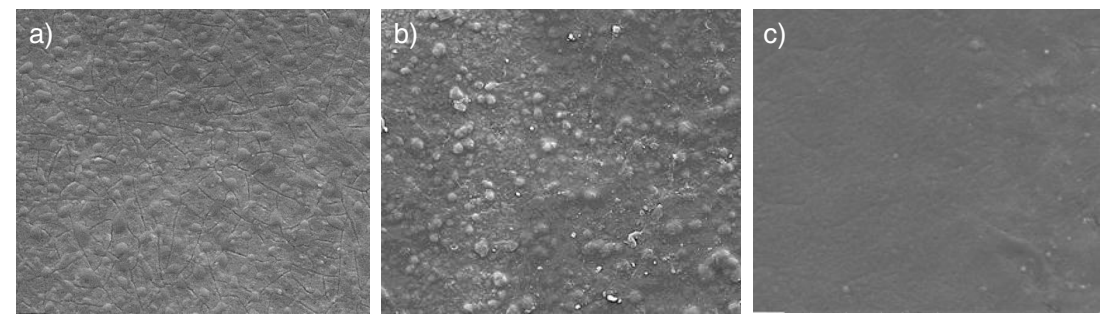

FIgURA 2. Micrografías SEM a $5000 \mathrm{X}$ para los recubrimientos con relación; a) 30\% PCL y 70\% Quitosano, b) $50 \%$ PCL y $50 \%$ Quitosano y c) $70 \%$ PCL y $30 \%$ Quitosano a $10000 X$.

se sobresaturó en el solvente a medida que éste fue evaporado, precipitando en la matriz como glóbulos o como una capa continua.

En la Figura 2c puede apreciarse la morfología superficial que presentaron los recubrimientos con relación $70 \%$ PCL y 30\% Quitosano. Los recubrimientos con relación 70-30 muestran una película polimérica completamente homogénea, continua, densa y sin presencia de la estructura porosa de quitosano y rugosidades en la superficie del sustrato.

\subsubsection{Caracterización por espectroscopia infrarroja (FTIR)}

La Figura 3 presenta los espectros FTIR ( $\%$ transmitancia) de las muestras cerámico-poliméricas. Se observan para los tres biopolímeros, las vibraciones correspondientes al estiramiento asimétrico y flexión del radical hidroxilo ( $\mathrm{R}-\mathrm{OH})$ a una longitud de onda de $3456 \mathrm{~cm}^{-1}$ y $1735 \mathrm{~cm}^{-1}$, respectivamente. De igual manera, en la misma longitud de onda del estiramiento del radical hidroxilo, aparece la vibración correspondiente al estiramiento asimétrico del grupo amino $\left(\mathrm{R}-\mathrm{NH}_{2}\right)$, el cual manifiesta a su vez un modo de flexión con una vibración a la longitud de onda de $1654 \mathrm{~cm}^{-1}$.

Se observa también la vibración correspondiente al estiramiento del radical R-OH del grupo carboxilo (R-COOH) en los ácidos orgánicos, a una longitud de onda de $2468 \mathrm{~cm}^{-1}$. El estiramiento de dicho radical es consecuencia de la existencia de remanentes del solvente empleado (ácido acético) en la matriz del recubrimiento (Pok et al., 2010). Además, aparecen vibraciones correspondientes a grupos carbonilo $(\mathrm{C}=\mathrm{O})$ a longitudes de onda de $1735 \mathrm{~cm}^{-1}$, correspondientes a la policaprolactona y ácido carboxílico.

En las vibraciones observadas en los espectros se encuentra la banda correspondiente al radical acetato (-COO) entre los $1238-1254 \mathrm{~cm}^{-1}$, debido a que el quitosano empleado no estaba completamente desacetilado. Así mismo, aparecen las vibraciones correspondientes al enlace O-Ti-O en el titanato de sodio, en la zona de longitudes de onda comprendidas entre $1041-1047 \mathrm{~cm}^{-1}$.

La falta de formación de nuevos enlaces sugiere que la interacción entre la policaprolactona y el quitosano en las películas es atribuida a un fenómeno de interacción física entre los biopolímeros por la unión por puentes de hidrógeno entre el grupo carbonilo de la policaprolactona y los grupos amino e hidroxilo del quitosano (Vera et al., 2010; Van der Schueren et al., 2012).

\subsection{Comportamiento de los recubrimientos en solución SBF}

En la Figura 4 se muestra la formación de precipitados de calcio y fósforo en las superficies poliméricas, posterior a los 8 días de inmersión en solución SBF. Transcurridos los 8 días de inmersión se observó la precipitación de fosfatos de calcio en la superficie de las películas poliméricas, lo cual manifiesta la capacidad de dichas películas 


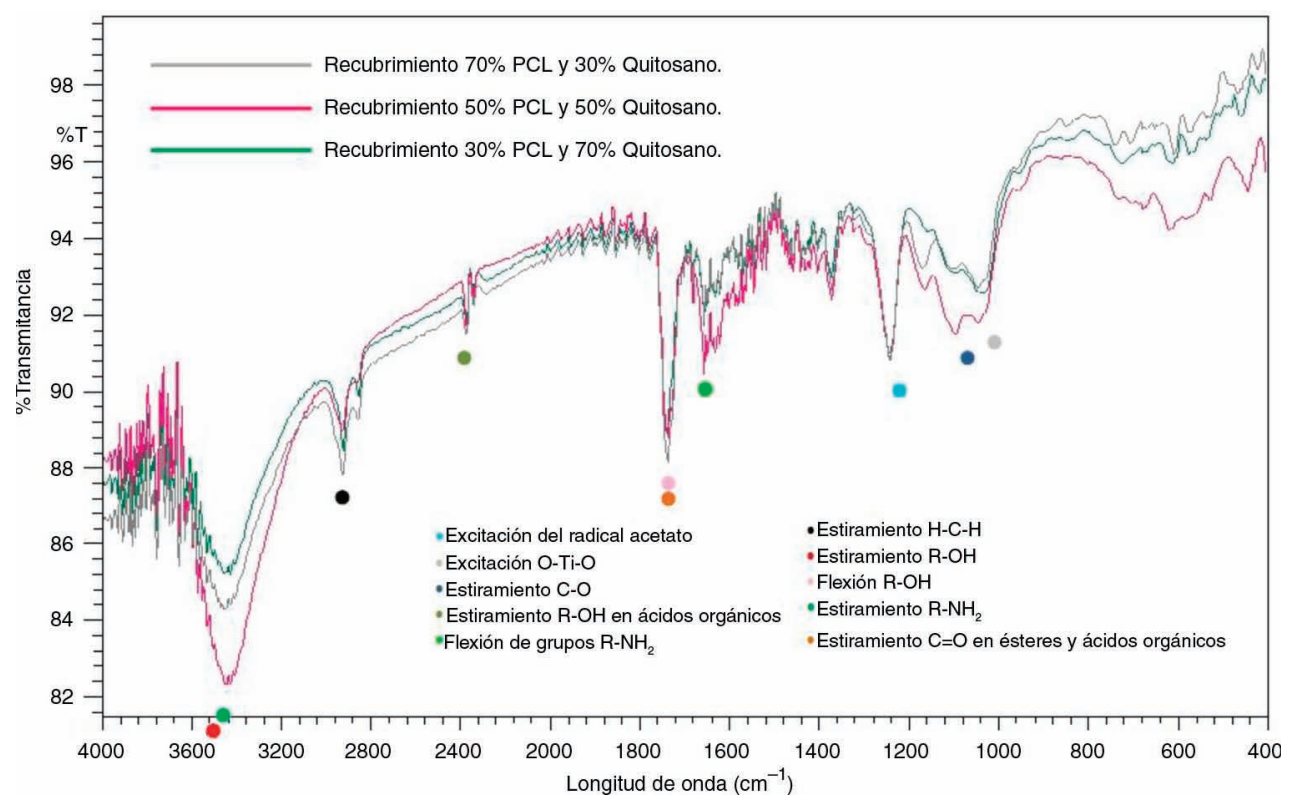

FIGURA 3. Espectros infrarrojos FTIR para las muestras cerámico-poliméricas con relaciones PCL-Quitosano: 30-70, 50-50 y 70-30.

para la adsorción de calcio; sin embargo la mayor cantidad de precipitados se observó en las muestras con relación PCL-Quitosano de 50-50, seguido por las muestras con relación PCL-Quitosano 70-30 y por último las películas para una relación de 30-70. La diferencia en la cantidad de precipitados es atribuida a la presencia del radical hidroxilo en las películas y al tipo de morfología (porosa o continua) de los recubrimientos.

La composición química de dichos precipitados pone de manifiesto que las relaciones $\mathrm{Ca} / \mathrm{P}$ son equivalentes a la relación existente entre el $\mathrm{Ca}$ y $\mathrm{P}$ en el fosfato dicálcico dihidratado $\mathrm{CaHPO}_{4} \cdot 2 \mathrm{H}_{2} \mathrm{O}$.

Las superficies de los recubrimientos con relación PCL-Quitosano de 50-50 y 70-30 después de los 8 días de inmersión en solución SBF, presentan un deterioro, debido a la pérdida de la matriz de quitosano y la aparición de grietas y glóbulos en las superficies de las películas, ocasionada por la degradación hidrolítica que manifiesta el quitosano presente, al interactuar con la solución SBF.

\subsection{Análisis electroquímico por Espectroscopia de Impedancia Electroquímica (EIE)}

El diagrama de Nyquist (Fig. 5), muestra el comportamiento electroquímico que presentan los recubrimientos poliméricos frente a la solución SBF. Se aprecia un incremento en los valores de impedancia de los recubrimientos, de manera proporcional al aumento de la cantidad de policaprolactona en las soluciones. Este comportamiento conlleva a que el recubrimiento con relación PCL-Quitosano de 70-30 presente el mayor valor de resistencia a la transferencia. En la Figura 6 se aprecia el diagrama de Bode para el módulo de la impedancia correspondiente a los recubrimientos evaluados. En la zona de bajas frecuencias $(0,01-1 \mathrm{~Hz})$, se observa un
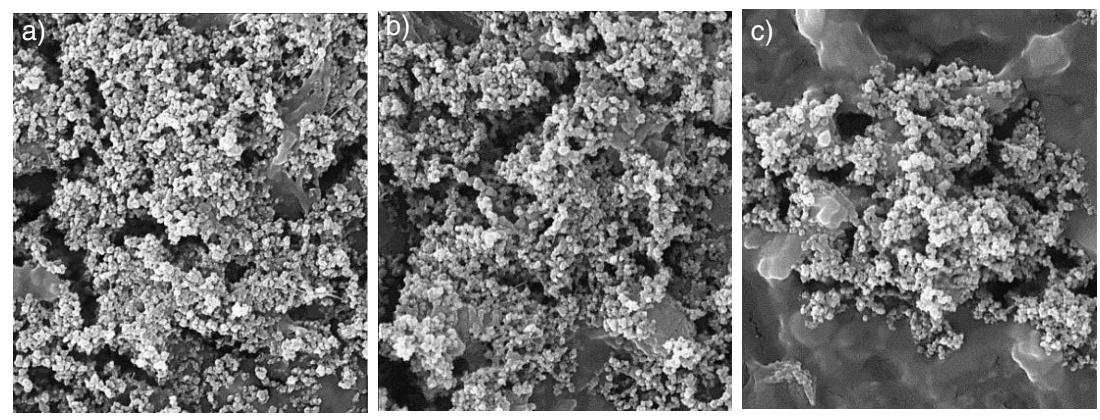

FIGURA 4. Micrografías SEM a 40.000X para los recubrimientos poliméricos de PCL-Quitosano después de 8 días de inmersión en fluido SBF: a) relación 30-70, b) relación 50-50 y c) relación 70-30. 


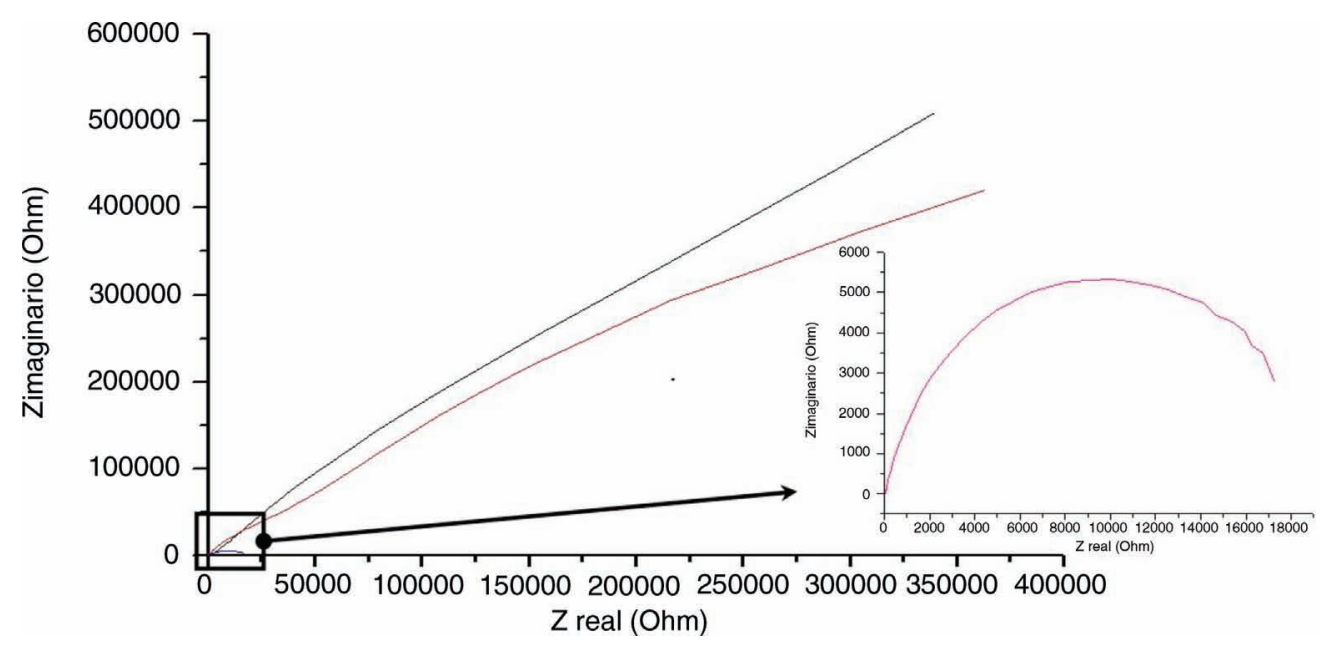

FIGURA 5. Diagramas de Nyquist para los recubrimientos con relación PCL-Quitosano: 30-70, 50-50 y 70-30 en solución SBF.

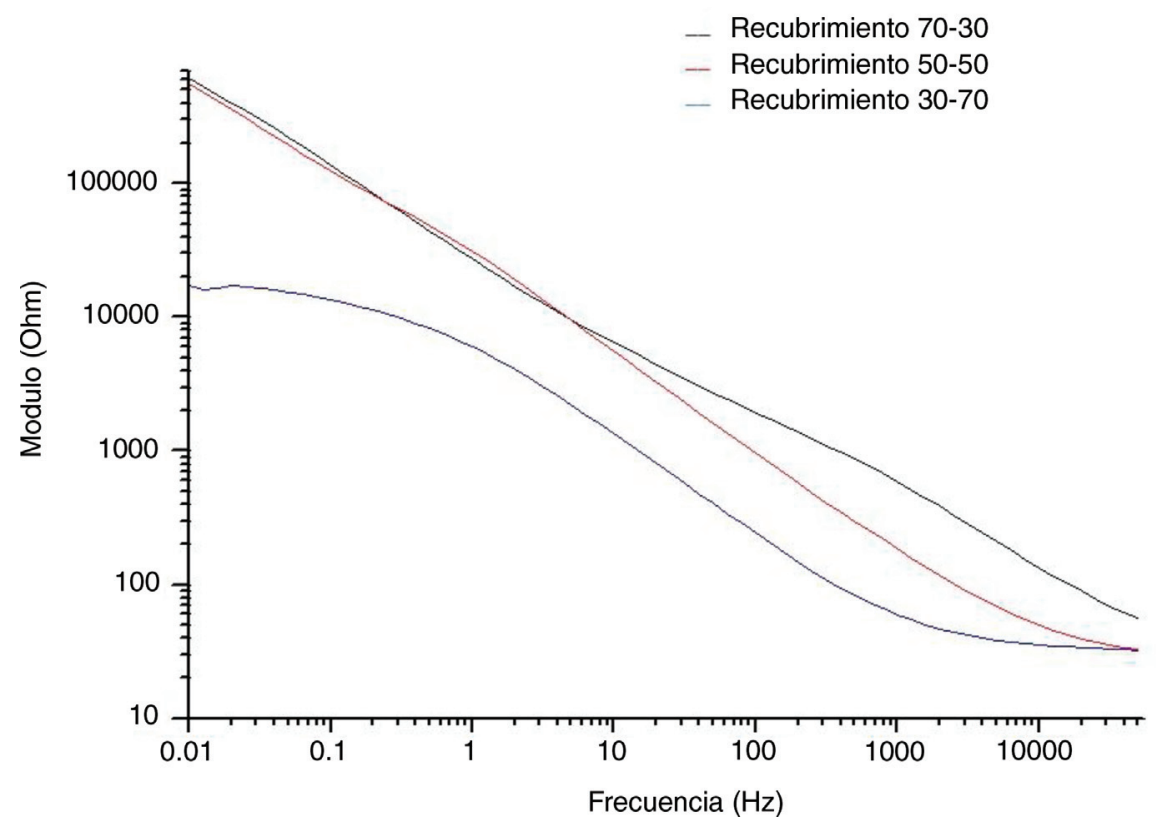

Figura 6. Diagramas de Módulo de impedancia vs. Log Frecuencia para los recubrimientos con relación PCL-Quitosano: 30-70, 50-50 y 70-30 en solución SBF.

comportamiento resistivo en las películas con una relación PCL-Quitosano de 70-30 y 50-50, al manifestar mayores valores del módulo de impedancia, gracias a la presencia de una película homogénea y densa en la superficie.

La Figura 7 presenta el diagrama de Bode para el ángulo de fase en función de la frecuencia (en escala logarítmica) en los recubrimientos poliméricos expuestos a la solución SBF. Se observa un cambio en el comportamiento de las películas, de capacitivo a resistivo, al presentarse una caída en los valores del ángulo de fase en la zona de frecuencias medias y altas para las tres relaciones poliméricas.
El cambio es debido a la presencia de porosidades en la interfase electrodo/electrolito, como consecuencia de los poros en la estructura del quitosano, así como a las faltas de zonas recubiertas con la mezcla polimérica.

La caída del ángulo de fase está relacionada con un proceso de adsorción de iones en la superficie, principalmente del ión calcio y el ión fosfato, generando una monocapa que obstruye parte de las porosidades existentes en el recubrimiento, aumentando la capacidad resistiva de la interfase (Cottis et al., 1999; Echeverria y Arroyave, 2003; Oshida, 2013; Quintero, 2013). 


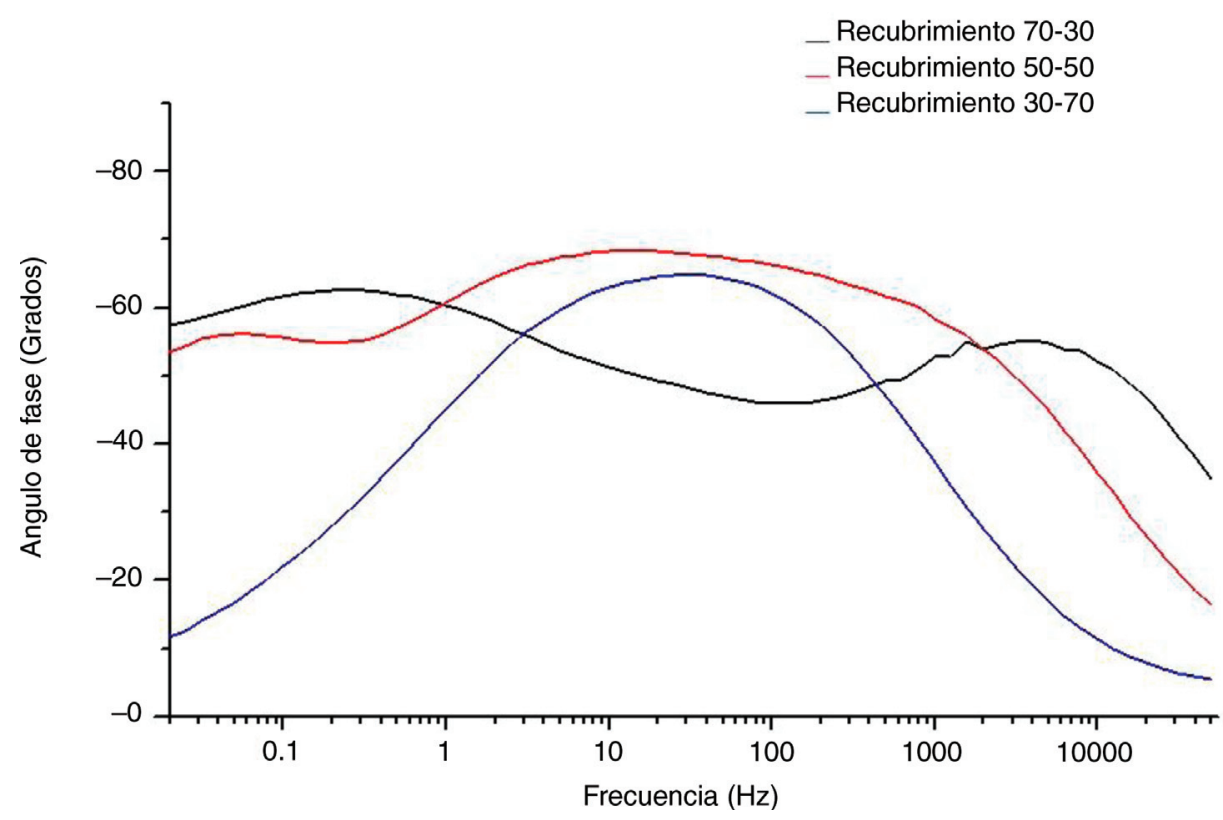

Figura 7. Diagramas de Ángulo de fase vs. Log Frecuencia para los recubrimientos con relación PCL-Quitosano: 30-70, 50-50 y 70-30 en solución SBF.

\subsection{Simulación del circuito equivalente propuesto}

En la Figura 8 se observan los circuitos eléctricos análogos propuestos, que relacionan los comportamientos observados en los espectros de impedancia, así como las características morfológicas observadas en los recubrimientos con relación PCL-Quitosano de $30-70,50-50$ y $70-30$.

Basándose en los circuitos planteados, se asociaron a los respectivos elementos del circuito, su componente físico en el sistema electrodo-electrolito, donde el primer elemento presente en ambos circuitos equivalentes $\left(\mathrm{R}_{\mathrm{s}}\right)$ corresponde a la resistencia óhmica que presenta el electrolito. En los circuitos para las relaciones PCL-Quitosano de 30-70 y 50-50 se observa una resistencia óhmica $R_{p}$ y un elemento de fase constante $C P E_{P}$, debido a la resistencia eléctrica y efecto dieléctrico, que generan las porosidades y defectos en la superficie del recubrimiento. De igual manera se asocia al sistema una resistencia eléctrica $\left(\mathrm{R}_{\mathrm{tc}}\right)$ que representa la resistencia a la transferencia de carga del electrodo (Ti6Al4V), y se presenta un elemento de fase constante $\mathrm{CPE}_{\mathrm{dl}}$ asociado a la formación de la doble capa eléctrica en dicha interfase.

En el caso del circuito correspondiente al recubrimiento con relación PCL-Quitosano de 70-30 se presentan las resistencias óhmicas $\mathrm{R}_{1}$ y $\mathrm{R}_{2}$, las cuales representan las resistencias al paso de la corriente del recubrimiento polimérico $70-30$ y del
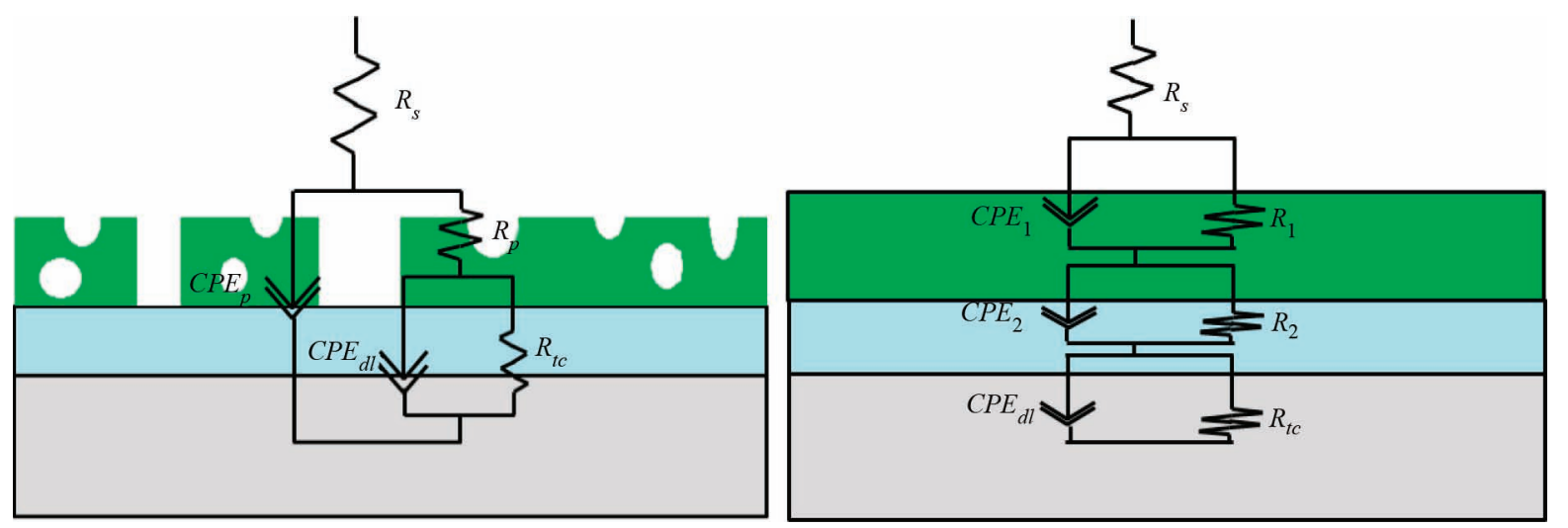

FIGURA 8. Representación de las interfases para los sistemas y su respectivos circuitos equivalentes: a) sistema compuesto por una subcapa interna densa y compacta y una capa externa porosa o defectuosa para las películas 30-70 y 50-50 y b) sistema compuesto por subcapas densas y compactas, una sobre la otra, para las películas 70-30. 
titanato de sodio, los cuales presentan sus respectivos elementos de fase constante $\mathrm{CPE}_{1} \mathrm{yCPE}_{2}$ dado el efecto dieléctrico que manifiestan las interfases del sistema de capas. De manera similar se asocian la resistencia a la trasferencia de carga $\left(\mathrm{R}_{\mathrm{tc}}\right)$ y el elemento de fase constante $\mathrm{CPE}_{\mathrm{dl}}$ debido a la formación de la doble capa eléctrica en interfase con el electrodo. Los valores obtenidos de los elementos resistivos en los circuitos equivalentes, correspondientes a la resistencia a la transferencia de carga, constataron que el aumento en la cantidad de PCL en la mezcla polimérica, causa un aumento en los valores de la resistencia a la transferencia de carga al presentarse valores de $2,9445 \times 10^{6}, 1,1452 \times 10^{6}$ y $8176 \mathrm{Ohm}$, para los recubrimientos poliméricos PCL-Quitosano de 70-30, 50-50 y 30-70, respectivamente.

\section{CONCLUSIONES}

Las cantidades de policaprolactona en los recubrimientos poliméricos mostraron un efecto en las características morfológicas en la superficie, debido a que promueven la formación de superficies heterogéneas, homogéneas, densas o matrices porosas.

La existencia de una mayor cantidad de grupos hidroxilo ( $\mathrm{R}-\mathrm{OH})$, así como la presencia de una matriz porosa de quitosano en los recubrimientos con relación PCL-Quitosano de 50-50, promovieron y facilitaron el proceso de adsorción de calcio y su precipitación, mostrando el mejor carácter bioactivo entre las relaciones poliméricas.

Las variaciones morfológicas en la superficie de los recubrimientos observados posterior a los 8 días de inmersión en SBF, corroboran el fenómeno de hidrólisis que experimenta el quitosano, lo cual le permite generar a esta clase de recubrimientos componentes metabolizables por las células, durante el proceso de osteosíntesis.

El comportamiento electroquímico observado en los espectros de impedancia permite corroborar los fenómenos relacionados con las características morfológicas de los recubrimientos poliméricos, así como la adsorción de especies iónicas, liberación de iones hacia el electrolito y formación de capas de fosfatos de calcio en ellos.

La capacidad de adsorción y precipitación del calcio y el fósforo, así como el proceso de degradación hidrolítica que muestran los recubrimientos de PCL-Quitosano, permiten a esta clase de recubrimientos biopoliméricos ser aplicados sobre materiales con poca bioactividad como el titanio, con el propósito de mejorar su reactividad química, y facilitar su utilización en la fabricación de implantes para regeneración y sustitución de tejidos óseos.

\section{AGRADECIMIENTOS}

Nuestros más sinceros agradecimientos a COLCIENCIAS, por la financiación de este proyecto bajo el contrato $\mathrm{N}^{\circ} 780-2011$ y al Grupo de Investigaciones en Corrosión (GIC) de la Universidad Industrial de Santander por su constante apoyo y colaboración.

\section{REFERENCIAS}

Bronzino, J. (2000). The biomedical engineering Handbook, Ed. CRS Taylor \& Francis, USA, pp. 37-44.

Cottis, R., Turgoose, S. (1999). Electrochemical Impedance and Noise, B.C. Syrett, Series Editor, USA, pp. 2-4.

Echeverria, A., Arroyave, C. (2003). Evaluación electroquímica de algunas aleaciones para implantes dentales del tipo titanio y acero inoxidable. Rev. Metal. 39, 174-181. http:// dx.doi.org/10.3989/revmetalm.2003.v39.iExtra.1116.

Estupiñan, H. (2011). Tesis Doctoral, Facultad de Ingenierías Fisicoquímicas, Universidad Industrial de Santander. Colombia.

Kim, H., Miyaji, F., Kokubo, T. (1997). Effect of heat treatment on apatite-forming ability of Ti metal induced by alkali treatment. J. Mater. Sci.- Mater. M. 8 (6), 341-347. http:// dx.doi.org/10.1023/A:1018524731409.

Multigner, M., Fernández-Castrillo, P., Ferreira-Barragans, S., González-Doncel G., González-Carrasco, J. (2009). Influencia del arenado de la aleación Ti6A14V en la dureza subsuperficial y estado de tensiones residuales. Rev. Metal. 45, 52-57. http://dx.doi.org/10.3989/revmetalm.0803.

Oshida, Y. (2013). Bioscience and Bioengineering of Titanium Materials, $2^{\circ}$ Ed. Elsevier, USA, pp. 35-85.

Paz, A., Martin, Y, Pazos, L., Parodi, M., Ybarra, G., González, J. (2011). Obtención de recubrimientos de hidroxiapatita sobre titanio mediante el método biomimético. Rev. Metal. 47, 138-146. http://dx.doi.org/10.3989/revmetalmadrid.1009.

Peón, E., Jimenez-Morales, A., Fernandez-Escalente, E., GarciaAlonso, M., Escudero, M., Galván, J. (2005). Recubrimientos de hidroxiapatita preparados mediante un proceso sol-gel. Rev. Metal. 41, 479-482. http://dx.doi.org/10.3989/ revmetalm.2005.v41.iExtra.1080.

Pok, S.W., Wallace, K., Madihally, S.V. (2010). In vitro characterization of polycaprolactone matrices generated in aqueous media. Acta Biomaterialia 6 (3), 1061-1068. http://dx.doi. org/10.1016/j.actbio.2009.08.002.

Quintero, A. (2013). Trabajo de grado, Facultad de Ingenierías Fisicoquímicas, Universidad Industrial de Santander, Colombia.

Reddy, A. (2011). Tesis Doctoral, Facultad en Ciencias de Ingeniería Química, Jawaharlal Nehru Technological University, India.

Tojal, C., Amigo, V., Calero, J. (2013). Fabricación y caracterización de aleaciones porosas de Ti y Ti6Al4V producidas mediante sinterización con espaciador. Rev. Metal. 49 , 20-30. http://dx.doi.org/10.3989/revmetalm.1206.

Van der Schueren, L., Steyaert, I., Schoenmaker, B., Clerckl, K. (2012). Polycaprolactone/Chitosan blend nanofibers electrospun from an acetic acid/formic acid solvent system. Carbohyd. Polym. 88, 1221-1226. http://dx.doi. org/10.1016/j.carbpol.2012.01.085.

Vera, M., Caridade, S., Alves, N., Mano, F.J. (2010). New poly(ecaprolactone)/Chitosan blend fibers for tissue engineering applications. Acta Biomaterialia 6 (2), 418-428. http:// dx.doi.org/10.1016/j.actbio.2009.07.012.

Xuanyong, L., Chub, K., Chuanxian, D. (2004). Surface modification of titanium, titanium alloys, and related Materials for biomedical applications. Mat. Sci. Eng. R. 47 (3-4), 49-121. http://dx.doi.org/10.1016/j.mser.2004.11.001. 